\title{
Zero-momentum modes and chiral limit in compact lattice QED *
}

\author{
I.L. Bogolubsky ${ }^{\mathrm{a}}$, V.K. Mitrjushkin ${ }^{\mathrm{a}}$, M. Müller-Preussker ${ }^{\mathrm{b}}$ and N.V. Zverev ${ }^{\mathrm{b}}$

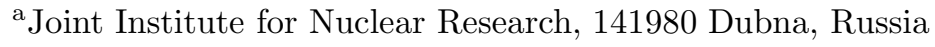 \\ ${ }^{\mathrm{b}}$ Humboldt-Universität zu Berlin, Institut für Physik, Invalidenstr. 110, D-10115, Germany
}

The influence of zero-momentum gauge modes on physical observables is investigated for compact lattice QED with dynamical and quenched Wilson fermions. Within the Coulomb phase, zero-momentum modes are shown to hide the critical behavior of gauge invariant fermion observables near the chiral limit. Methods for eliminating zero-momentum modes are discussed.

Lattice gauge theories allow to compute physical observables without gauge fixing. At the same time, more detailed information about nonperturbative properties of quantum fields can be extracted from gauge-dependent objects. But the straightforward application of iterative gauge fixing procedures leads to the appearance of gauge (Gribov) copies. For compact lattice QED in the Coulomb phase, such spurious gauge configurations disturb the correct perturbative behavior of both the photon and fermion correlators [1 [3]. Numerical [3 5] and analytical [6] investigations of this problem have shown the main excitations responsible for the occurence of such copies to be double Dirac sheets and zero-momentum modes. The former can be removed by usual, or periodic, gauge rotations. However, the removal of the latter requires constant, or nonperiodic, gauge transformations, which - for finite lattice size violate the invariance of the fermion matrix.

In this talk we present results of a study of the influence of zero-momentum modes in compact lattice QED within the physical (Coulomb) phase.

The action $S$ is given by

$$
\begin{aligned}
S & =S_{G}[U]+S_{F}[U, \bar{\psi}, \psi] \\
S_{G} & =\beta \sum_{x, \mu>\nu}\left(1-\operatorname{Re} U_{x \mu} U_{x+\hat{\mu}, \nu} U_{x+\hat{\nu}, \mu}^{*} U_{x \nu}^{*}\right),
\end{aligned}
$$

\footnotetext{
*Talk presented by N.V. Zverev
}

$S_{F}=\sum_{\mathrm{f}=1}^{N_{\mathrm{f}}} \sum_{x, y} \bar{\psi}_{x}^{\mathrm{f}} \mathcal{M}_{x y} \psi_{y}^{\mathrm{f}}$,

where $\mathcal{M}$ is the Wilson fermion matrix

$$
\begin{array}{r}
\mathcal{M}_{x y} \equiv \mathbf{1}_{\mathrm{D}} \delta_{x y}-\kappa \sum_{\mu}\left\{\left(\mathbf{1}_{\mathrm{D}}-\gamma_{\mu}\right) U_{x \mu} \delta_{y, x+\hat{\mu}}\right. \\
\left.+\left(\mathbf{1}_{\mathrm{D}}+\gamma_{\mu}\right) U_{y \mu}^{*} \delta_{y, x-\mu}\right\} .
\end{array}
$$

$U_{x \mu}=\exp \left(\mathrm{i} \theta_{x \mu}\right), \theta_{x \mu} \in(-\pi, \pi]$ denote the link variables. $\beta$ and $\kappa$ are the inverse square bare coupling and the hopping parameter, respectively. The gauge as well as the fermion field obey periodic boundary conditions (b.c.) except for the $x_{4}$ (time) direction, for which the fermion field will be taken either periodic or antiperiodic.

In what follows we consider both the quenched case simulated with a heat bath method and the dynamical case for $N_{\mathrm{f}}=2$ flavors studied with the hybrid Monte-Carlo method (HMC).

When studying gauge-dependent objects like gauge link correlators or the fermion propagator the Lorentz (or Landau) gauge fixing procedure is usually applied by iteratively maximizing the functional

$F[\Omega] \equiv \frac{1}{4 V} \sum_{x \mu} \operatorname{Re} U_{x \mu}^{\Omega} \longrightarrow \max$,

with $U_{x \mu}^{\Omega}=\Omega_{x} U_{x \mu} \Omega_{x+\mu}^{*}$ and $\Omega_{x} \in \mathrm{U}(1) . \quad V=$ $N_{s}^{3} \cdot N_{4}$ denotes the lattice volume. The gauge 


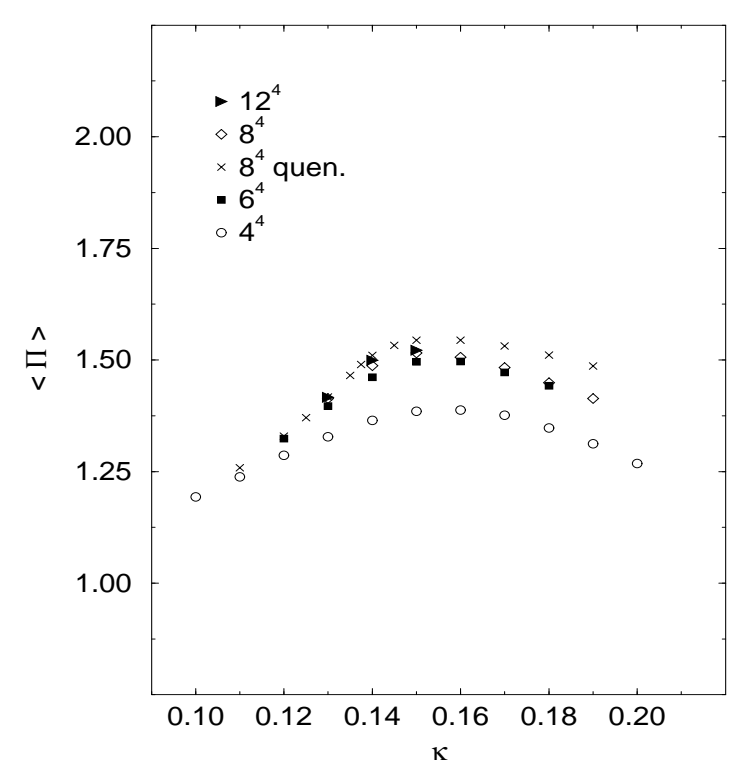

Figure 1. Pion norm as function of $\kappa$ for full (and quenched) compact QED with Wilson action at $\beta=1.1$ for various lattice sizes (data taken from [8]).

functional in most cases has many local extrema. Consequently the iterative maximization procedure can provide different gauge copies. But the 'best' or physically correct gauge copies are expected to be related to the global maxima.

In our recent paper [7] gauge variant fermion correlators in the quenched approximation have been calculated within the standard Lorentz gauge. It has been shown that these correlators behave strongly decaying, as if they were massive in the limit $\kappa \rightarrow \kappa_{c}(\beta)$. This effect has been shown caused by constant or zero-momentum modes of the gauge fields

$z_{\mu}[U] \equiv \frac{1}{V} \sum_{x} \theta_{x \mu}$.

Within the Lorentz gauge the zero-momentum modes do not allow to obtain the best gauge copy, i.e. the maximization of the functional $F[\Omega]$ de- fined in eq. (3) does not lead to its absolute maximum. Zero-momentum modes cannot be eliminated by usual (periodic) gauge transformations. The Lorentz gauge can only drive them into the interval $\left[-\pi / N_{\mu},+\pi / N_{\mu}\right]$ [7 which, however, appears to be sufficient to distort gauge-dependent observables. Increasing the lattice volume does not change the situation significantly.

In 때 an iterative Lorentz gauge fixing procedure combined with zero-momentum mode subtraction was proposed (ZML gauge). The zeromomentum mode suppression

$z_{\mu}[U]=0$

is achieved by appropriate non-periodic gauge transformation steps

$U_{x \mu} \rightarrow c_{\mu} U_{x \mu}, \quad c_{\mu} \in \mathrm{U}(1)$

following each Lorentz gauge step.

Within the ZML gauge the Lorentz functional (3) reaches its global maximum with high accuracy [4., 证. On the other hand, ZML gauge fixing leads to the correct perturbative behavior of gauge-dependent objects [4, [7]. In particular, the results are compatible with vanishing fermion masses near the chiral limit.

In this talk, instead of gauge variant objects we consider the gauge invariant - with respect to periodic gauge transformations - 'pion norm'

$$
\langle\Pi\rangle=\left\langle\frac{1}{4 V} \operatorname{Tr}\left(\mathcal{M}^{-1} \gamma_{5} \mathcal{M}^{-1} \gamma_{5}\right)\right\rangle \sim\left\langle\sum_{i} \frac{1}{\lambda_{i}^{2}}\right\rangle,
$$

where $\langle\cdots\rangle$ means the functional average with respect to the compact $U(1)$ gauge field variables. The $\lambda_{i}$ are the eigenvalues of $\gamma_{5} \mathcal{M}$. One expects $\langle\Pi\rangle$ to be a good indicator of the chiral limit at $\kappa \rightarrow \kappa_{c}(\beta)$, as some of the $\lambda_{i}$ are expected to tend to zero. It is worth noting that for periodic and time-antiperiodic b.c. for the fermion fields the averages $\langle\Pi\rangle$ strictly coincide.

However, the numerical study of fermionic observables like $\langle\Pi\rangle$ near the chiral limit does not reveal the critical properties as expected from lowest order and finite lattice size perturbation theory. This can be seen from the $\kappa$-dependence of the pion norm numerically computed at low $\beta$ values within the Coulomb phase [8] (see Fig. 11). 


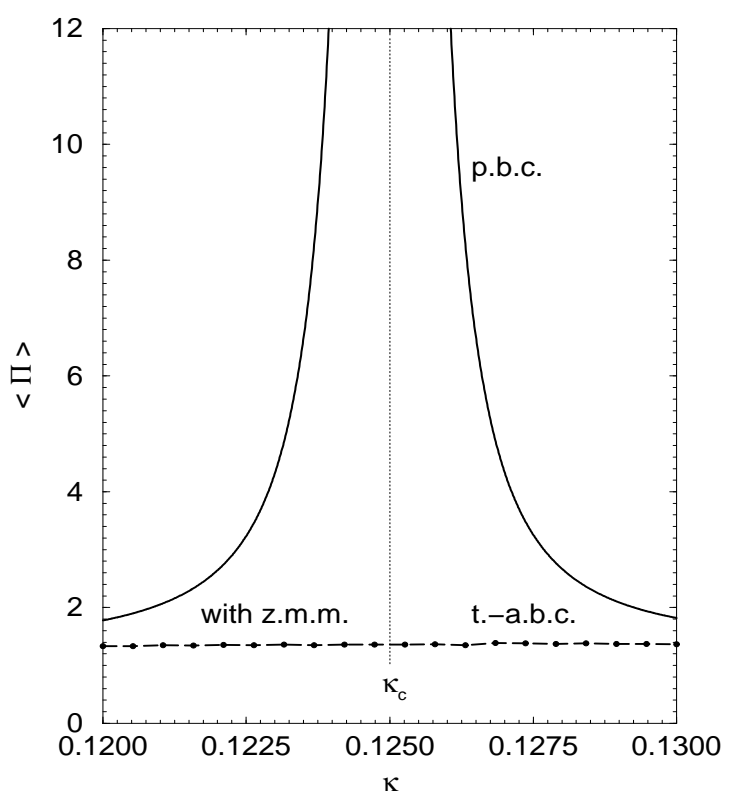

Figure 2. Pion norm in the free fermion case: without zero-momentum modes and in the zeromomentum mode background, lattice size $6^{4}$, periodic (p.b.c.), as well as time-antiperiodic (ta.b.c.) boundary conditions.

Its behavior is very smooth and no sign of any critical behavior is observed. The volume dependence of $\langle\Pi\rangle$ is rather weak, and there is no significant difference between the quenched and the dynamical case.

It is interesting to compare these results for $\langle\Pi\rangle$ with the free fermion case given by

$$
\begin{aligned}
\langle\Pi\rangle_{0}= & \frac{1}{V} \sum_{p}\left\{4 \kappa^{2} \sum_{\mu} \sin ^{2} \frac{2 \pi p_{\mu}}{N_{\mu}}+\right. \\
& \left.+\left(1-2 \kappa \sum_{\mu} \cos \frac{2 \pi p_{\mu}}{N_{\mu}}\right)^{2}\right\}^{-1},
\end{aligned}
$$

where the $p_{\mu}, \mu=1, \cdots, 4$ are integers except for time-antiperiodic b.c. causing $p_{4}$ to take half-

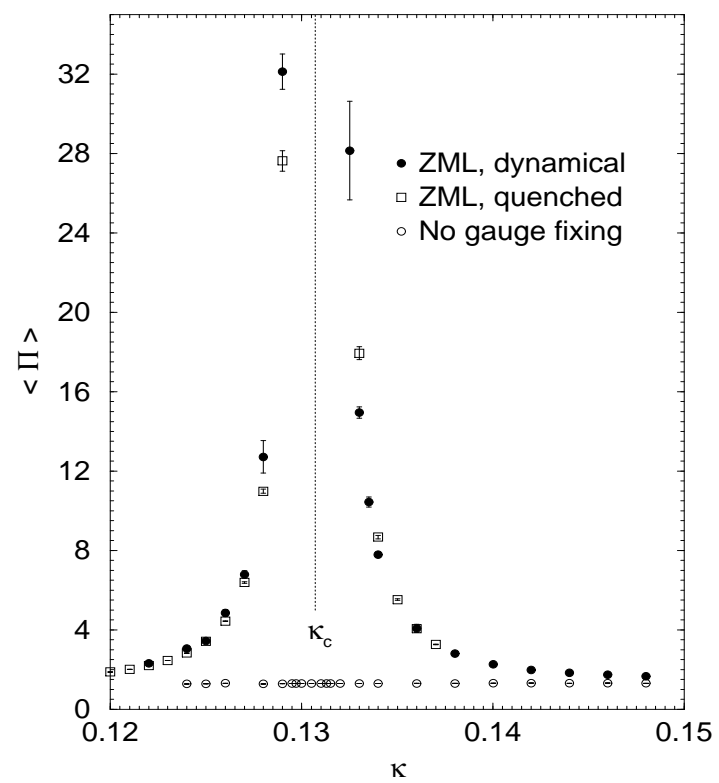

Figure 3. Pion norm as function of $\kappa$ redefined with ZML gauge $\left(\langle\Pi\rangle^{\prime}\right)$ for full and quenched QED, as well as without any gauge fixing $(\langle\Pi\rangle)$ for full QED; all data for $\beta=2.0$, lattice size $4^{4}$, periodic b.c.'s.

integer values. In Fig. 2 one can see the $\kappa^{-}$ dependence of $\langle\Pi\rangle_{0}$ calculated on a symmetric lattice $\left(N_{4}=N_{s}=6\right)$ for periodic and timeantiperiodic b.c. For periodic b.c. $\langle\Pi\rangle_{0}$ obviously gets singular at $\kappa=1 / 8$, whereas for timeantiperiodic b.c. the $\kappa$-dependence of $\langle\Pi\rangle_{0}$ becomes smooth for symmetric lattices. However, in the latter b.c. case $\langle\Pi\rangle_{0}$ develops a peak for strongly elongated lattices $\left(N_{4} \rightarrow \infty\right.$ with $N_{s}=$ fixed).

We are going to demonstrate that this drastic difference between $\langle\Pi\rangle$ (Fig. 1) and $\langle\Pi\rangle_{0}$ (Fig. 2) is due to constant or zero-momentum modes of the gauge fields $z_{\mu}[U]$ as defined in eq.(任).

For the free case $(\beta \rightarrow \infty)$ this can be easily demonstrated by integrating $\langle\Pi\rangle_{0}$ over constant modes. Both for periodic and time-antiperiodic 
b.c. it yields

$$
\begin{aligned}
<\Pi>= & \int_{-\pi}^{\pi} \frac{\mathrm{d}^{4} \phi}{(2 \pi)^{4}}\left\{4 \kappa^{2} \sum_{\mu} \sin ^{2} \phi_{\mu}+\right. \\
& \left.+\left(1-2 \kappa \sum_{\mu} \cos \phi_{\mu}\right)^{2}\right\}^{-1} .
\end{aligned}
$$

The latter expression is completely smooth in $\kappa$ and agrees with the former time-antiperiodic, free result for symmetric lattices (see Fig. 2).

Now let us consider the interacting case, i.e. for finite $\beta$-values. We redefine the pion norm such that zero-momentum modes become eliminated. Each gauge field configuration generated in the simulation is transformed subsequently by the ZML gauge procedure including non-periodic gauge transformations in order to suppress zeromomentum modes as described above. We average $\Pi$ with respect to the ensemble of transformed fields. Note that the fermionic part of the action is not invariant under constant gauge transformations (6). Therefore, we get a new average $\langle\Pi\rangle^{\prime}$ which differs from $\langle\Pi\rangle$.

In the following we choose periodic boundary conditions, because we expect from the free case that they lead to a more pronounced chiral behaviour than the time-antiperiodic ones.

In Figure 3 we show the dependence of the pion norm $\langle\Pi\rangle$ and $\langle\Pi\rangle^{\prime}$ on $\kappa$. One can see that for dynamical fermions (full circles) as well as for quenched fermions (boxes) the redefined observable $\langle\Pi\rangle^{\prime}$ has a sharp singularity near the point $\kappa_{c}=0.1307(1)$ for $\beta=2.0$ [7]. In contrast, the standard definition of the pion norm $\langle\Pi\rangle$ demonstrates a completely smooth behavior (open circles). We checked these results for $\langle\Pi\rangle^{\prime}$ also on larger lattices. For $\kappa$ approaching $\kappa_{c}$ the same critical behaviour is observed, whereas very close to and slightly above $\kappa_{c}$ the influence of an increasing number of very small fermionic eigenmodes leads to stronger fluctuations ('exceptional configurations'). The dynamical and quenched results resemble each other. This can be interpreted such that the zero-momentum modes - although removed from the observable $\langle\Pi\rangle^{\prime}-$ continue to dominate the fermionic determinant.

What about other (maybe simpler) methods to get rid off the constant modes (4) of the gauge fields? We have also considered the Polyakov line gauge invented in [3] which transforms the spatially averaged Polyakov line values into real numbers. We convinced ourselves that this nonperiodic gauge - without the necessity to employ the Lorentz gauge - leads to the same singular chiral behaviour of the pion norm as the ZML gauge.

Our main conclusion is that zero-momentum modes play an important rôle near the chiral limit of compact QED in the Coulomb phase, at least for finite lattices. They smooth out the critical chiral behaviour expected from lowest order perturbation theory for observables like the pion norm and the chiral condensate. In any case it is necessary to take the zero-momentum modes properly into account.

This research has been supported by the graduate college DFG-GK 271, by the RFRB grant 99-01-01230 and by the JINR Heisenberg-Landau program.

\section{REFERENCES}

1. A. Nakamura and R. Sinclair, Phys. Lett. B243 (1990) 396.

2. A. Nakamura and M. Plewnia, Phys. Lett. B255 (1991) 274.

3. V. G. Bornyakov, V. K. Mitrjushkin, M. Müller-Preussker and F. Pahl, Phys. Lett. B317 (1993) 596.

4. I. L. Bogolubsky, V. K. Mitrjushkin, M. Müller-Preussker and P. Peter, Phys. Lett. B458 (1999) 102.

5. I. L. Bogolubsky, L. Del-Debbio and V. K. Mitrjushkin, Phys. Lett. B463 (1999) 109.

6. V. K. Mitrjushkin, Phys. Lett. B389 (1996) 713; B390 (1997) 293.

7. I. L. Bogolubsky, V. K. Mitrjushkin, M. Müller-Preussker, P. Peter and N. V. Zverev, Phys. Lett. B476 (2000) 448.

8. A. Hoferichter, V. K. Mitrjushkin, M. MüllerPreussker, T. Neuhaus and H. Stüben, Nucl. Phys. B434 (1995), 358. 